\title{
Beyond the Shoulder: A Case Report of Regional Interdependence Treatment Approach in an Elite Level Tennis Player
}

\author{
Spencer W. Sullivan*, loonna Félix
}

Sports Medicine Institute, Hospital for Special Surgery, New York, NY, USA

\section{Article Info}

\section{Article Notes}

Received: August 03, 2021

Accepted: September 29, 2021

\section{*Correspondence:}

Mr. Spencer W. Sullivan, Sports Medicine Institute, Hospital for Special Surgery, New York, NY, USA; Telephone No: (317) 7752626; Email: spencer.sullivan44@gmail.com

${ }^{\circ} 2021$ Sullivan SW. This article is distributed under the terms of the Creative Commons Attribution 4.0 International License.

\section{Keywords:}

Regional interdependence

Rotator cuff tear

Tennis; Athlete

Conservative treatment

Physical therapy

Rehabilitation

\begin{abstract}
This clinical case presents an elite level 23-year-old female tennis athlete with a high-grade partial thickness tear of the supraspinatus with associated pain, dysfunction, and deficits in strength and range of motion (ROM) of her trunk and lower extremities. The purpose was to determine the efficacy of using a regional interdependence approach in the treatment of a partial supraspinatus tear with associated kinetic chain deficits in an elite level athlete. Following 24 physical therapy sessions, pain, mobility (ROM), strength, and function improved in the dominant shoulder in addition to further symmetry of the trunk and lower extremity due to a regional interdependence rehabilitation approach and returned to elite level of play. As a result of this case, it is important for clinicians to consider a comprehensive, global approach to patient rehabilitation following injury.
\end{abstract}

\section{Introduction}

It is common for overhead athletes to suffer from various shoulder injuries, including chronic shoulder overuse, rotator cuff tendinopathy and superior labral tear from anterior to posterior often associated with repetitive physical force and microtrauma to the shoulder of involved overhead movements ${ }^{1,2}$. Recent prevalence of shoulder injuries among tennis players of all levels range from $4-17 \%{ }^{2}$. Specifically, partial rotator cuff tendinitis and tearing (tendinopathy) are increasingly prevalent in high level athletes participating in overhead sports, often requiring conservative treatment measures prior to surgical intervention. Current standard clinical practice for shoulder conditions, including rotator cuff tendinopathy, does not include the assessment of kinetic chain as a region that may share movement deficits with the glenohumeral joint ${ }^{3,4}$. A broader approach of examining the whole body, including the kinetic chain can facilitate a clearer understanding of its ability to generate symptoms and underlie local impairments.

The regional interdependence approach (RIA) acknowledges and assesses compensatory strategies that may exist when one region of the body displays asymmetrical motion ${ }^{5}$. An RIA describes the treatment of adjacent as well as distant joints or areas of the body from the source of dysfunction of pain in one joint. In contrast, the traditional model tends to focus on a local mechanism or impairment as the source of the symptoms, and thus is limited in its ability to resolve symptoms. Currently, there is a paucity of literature on the efficacy of an RIA in rehabilitation (i.e., a global rather than shoulder-intensive approach) of elite, overhead athletes with shoulder tendinopathy. The purpose of this case study was to examine the efficacy of a conservative RIA in rehabilitation on an 
elite tennis athlete with a high-grade partial thickness tear of the supraspinatus with associated deficits to the kinetic chain. We hypothesized successful treatment and full return to play following this RIA to treatment.

\section{Materials and Methods}

Clinical examination and radiographic imaging, both X-ray and magnetic resonance imaging (MRI), were assessed. Clinical criteria included pain (assessed as either as positive or negative with each exercise), strength (variable based on exercise type), range of motion (degrees), flexibility (modified Thomas Test) and subjective patient experience via the Quick DASH ${ }^{6}$. A score improvement of at least $>20$ was indicative of a positive responder with successful outcome ${ }^{7,8}$. MRI was assessed to identify degree of tearing, partial versus full-thickness tearing. Informed consent was obtained under the Hospital for Special Surgery Institutional Review Board (IRB) to present this clinical case.

\section{Clinical Case Description}

\section{History}

A 23-year-old elite female tennis athlete presented to the ambulatory care center with chronic right shoulder pain and dysfunction, indicative of rotator cuff tendinopathy. She was right hand dominant, measuring $180 \mathrm{~cm}$ and weighing $63.5 \mathrm{~kg}$, and has been playing tennis since the age of six. She reported worse symptoms three months prior to evaluation with associated dysfunction in her serving, overhead and forehand moves as well as activities of daily living. She completed physical therapy and returned to competition eight months after initial evaluation. Previous treatment consisted of intermediate phase local strengthening, motor control and stability training at the shoulder/scapular level, including global treatment focus on kinetic linking and movement patterning. Following the Challenger season, the athlete experienced further dysfunction and reported to the ambulatory care center once again for evaluation. She was diagnosed with highgrade partial thickness supraspinatus tear with internal impingement. Surgical treatment was not recommended by the physician as the patient was currently competing at a high level, on tour at the Challenger level, a "minor league," or lower level of professional tennis. A conservative course of treatment included a corticosteroid injection to the right shoulder, followed by physical therapy to treat pain and dysfunction.

\section{Physical Examination}

Upon evaluation, right shoulder pain and reduced strength was associated with manual muscle testing in external rotation $(3+/ 5)$, flexion (4-/5 strength), and abduction $(3+/ 5)$ alongside pain when testing the lower and middle trapezius (both 3/5). Range of motion deficits in internal range of motion at $90^{\circ}$ were found in the right shoulder $\left(40^{\circ}\right.$ compared to $80^{\circ}$ in the left) as well as end range pain in flexion and abduction in the right shoulder. The patient's assessment revealed local glenohumeral deficits in addition to deficits in her movement patterns and lower body strength/flexibility, including manual muscle testing asymmetry in hip abduction and extension (both 3+/5 compared to 4-/5 on the left) and reduced hold time in a single leg bridge ( 5 seconds on the right compared to 10 seconds on the left) with a pelvic drop. The 2-joint Thomas Test was performed on the right leg measuring $60^{\circ}$ compared to $70^{\circ}$ on the left leg (Table 1). Rehabilitation consisted of evaluation and treatment of the shoulder and kinetic chain, including the trunk and lower extremities.

\section{Treatment Approach}

An RIA approach was selected to comprehensively assess the contributing sources of this patient's shoulder injury. Treatment included modalities, soft tissue mobilization, local treatment of shoulder impairments (Glenohumeral Internal Rotation Deficit [GIRD] and Total Range of Motion [TROM], scapular dyskinesis). The RIA treatment approach of the kinetic chain involved functional-based therapeutic exercise of proximal hip musculature and core as well mobility of the trunk and anterior hip sling. Thorax, lower extremity (gluteal firing, timing, and endurance) impairments in addition to tennis mechanics were addressed in correcting shoulder dysfunction and return to competition. Succinctly, treatment consisted of movement linkage and transfer of power between the shoulder, core, hip and kinetic chain. In addition, equipment and video analysis of the patient's tennis serve and groundstrokes were performed.

\section{Outcomes}

The patient was treated for a total of 24 sessions in four months. Range of motion in internal rotation increased from $40^{\circ}$ to $55^{\circ}$ in the right shoulder and no end range pain was noted in flexion and abduction. Additionally, no pain was perceived in manual muscle testing alongside increased strength in external and internal rotation (both $5 / 5)$, flexion (5/5), abduction (5/5) and middle and lower trapezius (both $4+/ 5$ ). From the RIA approach, the patient's right hip additionally gained strength improving to $4 / 5$ in hip abduction and $4+/ 5$ for hip extension. Additionally, the Thomas Test was repeated for the right lower extremity, increasing from $60^{\circ}$ to $70^{\circ}$ post-rehabilitation. The Quick Disabilities of the Arm, Shoulder and Hand Score (DASH) and Quick DASH Sports Module decreased substantially over the course of rehabilitation from 42.5 to 4.5 and 56.3 to 12.3 , respectively (Table 1 ). Following rehabilitation, the athlete returned to her previous level of play at the 
Table 1: Clinical Characteristics of Pre- and Post- Rehabilitation

\begin{tabular}{|c|c|c|}
\hline Test Performed & Initial Evaluation & Discharge Date \\
\hline \multicolumn{3}{|l|}{ Shoulder Active Range of Motion } \\
\hline ER at $90 / 90^{\circ}$ & Right $118^{\circ}$ & Right $118^{\circ}$ \\
\hline IR at $90 / 90^{\circ}$ & Right $40^{\circ}$ & Right $55^{\circ}$ \\
\hline \multirow{2}{*}{ Flexion } & Right $\sim 175^{\circ}$ & Right $\sim 175^{\circ}$ \\
\hline & $(+)$ end range pain. & (-) end range pain \\
\hline \multirow{2}{*}{ Abduction } & Right $\sim 170^{\circ}$ & Right $\sim 175^{\circ}$ \\
\hline & $(+)$ end range pain. & (-) end range pain \\
\hline \multicolumn{3}{|l|}{ Shoulder Manual Muscle Test } \\
\hline ER $90 / 90^{\circ}$ & $3+/ 5(+)$ pain & $5 / 5(-)$ pain \\
\hline IR $90 / 90^{\circ}$ & $4-/ 5$ & $5 / 5(-)$ pain \\
\hline Flexion & 4-/5 (+) pain & $5 / 5(-)$ pain \\
\hline Abduction & $3+/ 5(+)$ pain & $5 / 5(-)$ pain \\
\hline \multirow{2}{*}{ Middle Trapezius } & Right $3 / 5$ & Right $4+/ 5$ \\
\hline & $(+)$ pain & $(-)$ pain \\
\hline \multirow{2}{*}{ Lower Trapezius } & Right $3 / 5$ & Right $4+/ 5$ \\
\hline & $(+)$ pain & (-) pain \\
\hline \multicolumn{3}{|l|}{ Hip/Knee Manual Muscle Test } \\
\hline Hip Abduction & Right $3+/ 5$ & Right $4 / 5$ \\
\hline Hip Extension & Right $3+/ 5$ & Right $4+/ 5$ \\
\hline Single Leg Bridge Hold & Left $10 \sec (+)$ pelvic drop & -- \\
\hline \multicolumn{3}{|l|}{ Hip/Knee Flexibility } \\
\hline \multirow{2}{*}{ Thomas Test } & Right $(+) 2$ joint $\mathrm{KF} \sim 60^{\circ}$ & \multirow{2}{*}{ Right $(+) 2$ joint $K F \sim 70^{\circ}$} \\
\hline & Left $(+) 2$ joint KF $\sim 70^{\circ}$ & \\
\hline \multicolumn{3}{|l|}{ Outcome Measures } \\
\hline Quick DASH & 42.5 & 4.5 \\
\hline Quick DASH Sports Module & 56.3 & 12.3 \\
\hline
\end{tabular}

Abbreviations: WFL, within functional limits; ER, External Rotation; IR, Internal Rotation; --, not obtained

Challenger level without pain, increased her serving velocity, and won her first International Tennis Federation point. No adverse or unanticipated events occurred in this case.

\section{Discussion}

Functional improvements were reported following a 24-session, 4-month RIA rehabilitation program for a partial supraspinatus tear. Prior to rehabilitation, the tennis athlete experienced pain at end range of motion (flexion and abduction) of the shoulder as well as during manual muscle testing of the shoulder with noticeable atrophy. Following the RIA rehabilitation program, the patient no longer experienced pain during manual muscle and range of motion testing. Additionally, significant patient reported outcome improvements were achieved in Quick DASH score (42.5 to 4.5) and the DASH Sports Module (56.3 to 12.3). At discharge, the patient demonstrated optimal scapular improvement and successful return to competition without pain or apprehension (serving speeds increased form $70 \mathrm{mph}$ to $>90 \mathrm{mph}$ ).

For elite female tennis athletes arthroscopic intervention is usually the last resort with low rates of return to previous level of play ${ }^{9,10}$. Therefore, conservative methods, such as rehabilitation are commonly employed for tennis athletes hoping to return to sport after a shoulder injury. An interval return program is one of many rehabilitation techniques designed to return an athlete back to their level of play ${ }^{11}$. However, to date, there is a paucity of research on conservative RIA rehabilitation for elitelevel tennis athletes. Former studies have examined the effects of regional interdependence on the cervical and thoracic spine ${ }^{12,13}$. Specifically, a recent systematic review analyzed the role of regional interdependence within baseball athletes. Not only were lower extremity deficits risk factors for upper extremity injury and/or pain, but improvements to these deficits reduced the incidence of upper extremity pain and dysfunction in young baseball athletes $^{14}$. Similarly, a cross-sectional study of 2,215 overhead athletes found that upper extremity pain was more prevalent in young athletes that had concomitant back, hip, knee and foot pain ${ }^{15}$. Prior to treatment, our patient demonstrated a break in the kinetic chain (lower extremity deficits) which may have been associated with the indicated supraspinatus tear. Following an RIA rehabilitation program, the athlete showed improvements in both upper and lower extremity range of motion, strength, and function. 


\section{Limitations}

This case report is certainly not without limitations. This single subject undergoing rehabilitation with an RIA is inherently limited in the conclusions that can be drawn. This case demonstrated the clinical usefulness of an RIA to return this athlete, with her specific history, to elite level of play in tennis. However, it is undetermined whether similar athletes with similar situations can avoid surgical intervention using an RIA. Additionally, there is no way to determine return to play at an elite level was associated with the regional interdependence component of this rehabilitation plan. Thus, causal analysis cannot be determined due to several potential confounding factors that were inherently present. Finally, it is important to note that previous failed nonoperative intervention as well as inadequate follow-up to the present could have skewed the results of this case report.

\section{Conclusion}

Injuries to the upper extremity are common in high level overhead sports, such as tennis. A regional interdependence approach was taken in this athlete's rehabilitation program, targeting the injury to the rotator cuff while further improving deficits in the kinetic chain and lower extremity. This case supports the efficacy of utilizing an RIA rehabilitation program to aid conservative treatment prior to return to competition.

\section{Funding}

This case report has no sponsorship support.

\section{Conflict of Interest}

Spencer Sullivan and Ioonna Félix have no conflicts of interest to declare.

\section{References}

1. Abrams GD, Renstrom PA, Safran MR. Epidemiology of musculoskeletal injury in the tennis player. Br J Sports Med. 2012; 46(7): 492-8.

2. Alrabaa RG, Lobao MH, Levine WN. Rotator Cuff Injuries in Tennis Players. Curr Rev Musculoskelet Med. 2020; 13(6): 734-47.
3. Guzowski K, Stolarczyk A, Czyrny Z, et al. Assessment of ultrasonography as a diagnostic tool in shoulder pain and alterations in glenohumeral range of motion in tennis players. Wideochirurgia Inne Tech Maloinwazyjne Videosurgery Miniinvasive Tech. 2019; 14(1): 114-25

4. Keller RA, De Giacomo AF, Neumann JA, et al. Glenohumeral Internal Rotation Deficit and Risk of Upper Extremity Injury in Overhead Athletes: A Meta-Analysis and Systematic Review. Sports Health. 2018; $10(2): 125-32$.

5. Wainner RS, Whitman JM, Cleland JA, et al. Regional interdependence: a musculoskeletal examination model whose time has come. J Orthop Sports Phys Ther. 2007; 37(11): 658-60.

6. Peeler JD, Anderson JE. Reliability Limits Of The Modified Thomas Test For Assessing Rectus Femoris Muscle Flexibility About The Knee Joint. J Athl Train. 2008; 43(5): 470-6.

7. Garcia AN, Thigpen CA, Lake AD, et al. Do older adults with shoulder disorders who meet the minimal clinically important difference also present low disability at discharge? An observational study. Braz J Phys Ther. 2020; 24(2): 152-60.

8. St-Pierre C, Desmeules F, Dionne CE, et al. Psychometric properties of self-reported questionnaires for the evaluation of symptoms and functional limitations in individuals with rotator cuff disorders: a systematic review. Disabil Rehabil. 2016; 38(2): 103-22.

9. Young SW, Dakic J, Stroia K, et al. Arthroscopic Shoulder Surgery in Female Professional Tennis Players: Ability and Timing to Return to Play. Clin J Sport Med Off J Can Acad Sport Med. 2017; 27(4): 357-60.

10. Ryösä A, Laimi K, Äärimaa V, et al. Surgery or conservative treatment for rotator cuff tear: a meta-analysis. Disabil Rehabil. 2017; 39(14): 1357-63.

11. Félix I, Dines D, Dines J. Interval Return to Play Programs for the Tennis Athlete. Curr Rev Musculoskelet Med. 2021; 14(2): 185-91.

12. McDevitt A, Young J, Mintken P, et al. Regional interdependence and manual therapy directed at the thoracic spine. J Man Manip Ther. $2015 ; 23(3): 139-46$.

13. Kahlaee $\mathrm{AH}$, Ghamkhar L, Nourbakhsh MR, et al. Strength and range of motion in the contralateral side to pain and pain-free regions in unilateral chronic non-specific neck pain patients. Am J Phys Med Rehabil. 2020; 99(2): 133-141.

14. Deal MJ, Richey BP, Pumilia CA, et al. Regional Interdependence and the Role of the Lower Body in Elbow Injury in Baseball Players: A Systematic Review. Am J Sports Med. 2020; 48(14): 3652-60.

15. Sekiguchi T, Hagiwara Y, Momma H, et al. Coexistence of Trunk or Lower Extremity Pain with Elbow and/or Shoulder Pain among Young Overhead Athletes: A Cross-Sectional Study. Tohoku J Exp Med. 2017; 243(3): 173-8. 